\title{
HER MAJESTY THE QUEEN TO ATTEND OFFICIAL CEREMONY FOR NEW BIRMINGHAM DENTAL HOSPITAL AND SCHOOL OF DENTISTRY
}

Her Majesty The Queen, accompanied by His Royal Highness The Duke of Edinburgh, officially unveiled a commemorative plaque at an official ceremony on Thursday 19 November to open the new Birmingham Dental Hospital and School of Dentistry at Pebble Mill, Edgbaston.

Her Majesty met clinicians, researchers and students during a guided tour of the state-of-the-art facilities.

The hospital, managed by Birmingham Community Healthcare NHS Trust, will be the new home of specialist dental treatment for West Midlands' patients and also houses the University of Birmingham's School of Dentistry, strengthening a partnership delivering dental health and education in the city since 1880 .

The four-storey building will provide a range of high quality clinical dental services for the public in one wing, while a second wing will offer world-class research facilities and a modern learning environment for more than 600 undergraduate and postgraduate

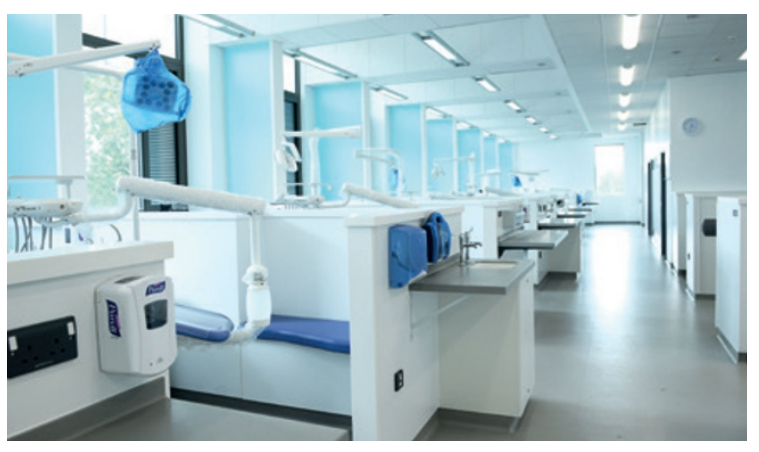

students and trainees each academic year.

With a total cost of $£ 50 \mathrm{~m}$, the development is a key element of the new Edgbaston Medical Quarter and further enhances the city's reputation as a hub of medicine, learning and life sciences.

University staff and students will enjoy the benefits of being located much closer to the main Edgbaston campus and medical school, allowing for closer integration of facilities.

The development represents the first integrated, stand-alone dental hospital and dental school to be built in the UK for almost 40 years. The development replaces the 50-year-old dental hospital at St Chad's Circus.

Tracy Taylor, chief executive of Birmingham Community Healthcare NHS Trust said: "The first class public facilities at the new Birmingham Dental Hospital and School of Dentistry symbolise everything we want to achieve as a provider of high quality healthcare services.
'We are committed to working with partners to provide first class care and treatment for all the communities we serve in modern, accessible locations with high quality facilities.

'This is a real success story that shows how partners from different sectors can together deliver valuefor-money infrastructure projects that meet the needs and expectations of public and practitioners.'

Professor Philip Lumley, head of the School of Dentistry at the University of Birmingham, said: 'This new building will be a stateof-the-art home for our world-class teaching and research.

'Not only will the people of the city and region be able to access firstclass treatment within this building, but we will be training the dental team of the future right here as well.

'This educational offering combined with the ground-breaking research which is carried out by our staff will allow us to develop further innovations to underpin the oral and dental health of the region and beyond.'

\section{AND FINALLY ...}

People over the age of 50 and women of all ages are more likely to have a lower weight if they regularly engage in high impact walking compared to doing another vigorous activity, according to a new publication from the London School of Economics and Political Science (LSE).

There is a similar finding for people on low incomes. The conclusions are likely explained by walking being zero cost and convenient for even the busiest of schedules.

Dr Grace Lordan, a specialist in health economics who led the research, examined reported physical activity levels from the annual Health Survey for England (HSE) from 1999 to 2012. In particular, she focused on activities which increased heart rate and caused perspiring. Individuals reported on the number of periods they engaged in 30 minutes or more of:
- Walking at a fast or brisk pace

- Moderate-intensity sports or exercise, such as swimming, cycling, working out at the gym, dancing, running, football/rugby, badminton/tennis, squash, and exercises including press-ups and sit-ups

- Heavy housework, such as moving heavy furniture, walking with heavy shopping, scrubbing floors

- Heavy manual activities, such as digging, felling trees, chopping wood and moving heavy loads.

She then analysed nurse collected data on body mass index (BMI) and waist circumference (WC) measurements and found that men and women who regularly walked briskly for more than 30 minutes had lower BMIs and smaller waists than those who engaged in regular sports/exercise.

The article explains that although the government recommends that adults should do 150 minutes or more of moderate-intensity physical activity every week, there is no guidance as to which activity is the most effective. The authors estimated that almost $80 \%$ of the UK population is not meeting the government-specified targets. This costs the NHS almost $€ 1$ billion a year, with much of these costs being attributed to the effects of obesity.

It concludes by calling for a campaign to promote walking as a potentially effective way to tackle obesity rather than public health messages on healthy diets: 'The results thus provide an argument for a campaign to promote walking...Focus on physical activity is less controversial as it would not be subject to political lobbying as is the case for 'fat' tax and other policies that aim to change consumption of junk foods in a person's diet.'

It adds: 'Given the obesity epidemic and the fact that a large proportion of people in the UK are inactive, recommending that people walk briskly more often is a cheap and easy policy option. Additionally, there is no monetary cost to walking so it is very likely that the benefits will outweigh the costs. It has also been shown by the same authors that walking is associated with better physical and mental health. So, a simple policy that 'every step counts' may be a step towards curbing the upward trend in obesity rates and beneficial for other health conditions.' 